\title{
Qubit Hamiltonian identification: A symmetry-preserving observer-based approach *
}

\author{
S. Bonnabel ${ }^{*}$ M. Mirrahimi ${ }^{* *}$ P. Rouchon ${ }^{* * *}$ \\ * Department of Electrical Engineering and Computer Science (Montefiore \\ Institute B28), University of Liège, Belgium (e-mail: \\ bonnabel@montefiore.ulg.ac.be) \\ **Inria Rocquencourt (e-mail: mazyar.mirrahimi@inria.fr) \\ *** Ecole des Mines de Paris, Centre Automatique et Systèmes, (e-mail: \\ pierre.rouchon@ensmp.fr)
}

\begin{abstract}
We consider a pure two-state quantum system illuminated by two lasers. A photo-detector captures the fluorescence of the system. We build an invariant observer which yields a (local) estimation of the wave function (or density matrix) and the two key parameters (laser de-tuning and the atomlaser coupling strength) of the hamiltonian. The design exploits the symmetries of the system and can be interpreted geometrically. The convergence proof is based on averaging arguments. Simulation with noise illustrates the robustness of the obtained estimation algorithm.
\end{abstract}

Keywords: Quantum systems, symmetries, asymptotic observers, estimation, nonlinear systems

\section{INTRODUCTION}

In this paper, we consider a quantum system for which we would like to identify some parameters. This estimation problem has already been addressed with interesting theoretical contributions concerning well-posedness and identifiability (see, e.g., Claude Le Bris et al. [2007] and the reference herein) and the development of estimation algorithms robust with respect to the noises and the modeling errors. Here, we consider as a simple quantum system, a two level system described by a Schrödinger dynamics and a time-continuous population measurement. We propose an algorithm based on nonlinear asymptotic observer techniques preserving the symmetries (Bonnabel et al. [2006]) to estimate the system parameters. As far as we know, such recent techniques have not been applied to tackle this problem and this paper illustrates their potential interest. Since the observer design exploits the physical symmetries (invariance with respect to the frame-change), the filter equation admits a natural physical and geometrical interpretation that can be extended to higher dimensions.

The physical setup consists of an ensemble of identically prepared systems undergoing the same dynamics: for instance, dilute mono-atomic gases. They are very simple systems, in the sense their constituents (atoms), are perfectly identical and interact very weakly with each other. Atoms in such gases can be considered as perfect quantum systems, with a sequence of discrete energy states labeled $|i\rangle$, for $i \in \mathbb{N}$, with increasing energies $E_{i}=\hbar \omega_{a, i}$ depending only on the atomic species considered. In order to measure the population of the ground state (i.e, the state of the lower energy), the system is illuminated with coherent light (a first laser) whose frequency is close to the transition frequency corresponding to the energy gap $E_{j}-E_{0}=\hbar\left(\omega_{a, j}-\omega_{a, 0}\right)$ of a very unstable excited state (i.e, having a very short lifetime) to the ground state transition. It

\footnotetext{
* This work was supported in part by the "Agence Nationale de la Recherche" (ANR), Projet Blanc CQUID number 06-3-13957.
}

generates a transition $|0\rangle \rightarrow|j\rangle$ for a part of the population illuminated from the ground state to the excited state, which spontaneously decays to the ground state emitting a photon. The measurement of the number of photons emitted is then directly proportional to the population of the ground state $|0\rangle$. Suppose there is also another laser whose frequency is close to the transition frequency of another excited state $|e\rangle$ to the ground state $|0\rangle$. The lifetime of the transition between the two latter states is supposed to be much longer than the previous one. To a first approximation the dynamics of the twostate system (ground state $|0\rangle$ and excited state $|e\rangle$ having the longest lifetime) is described by a Schrödinger equation. We assume that some parameters are not well known: $\Delta$ which is the difference between the second laser frequency and the atomic transition frequency, $\mu$ which is the atom-laser coupling strength and characterizes the Rabi frequency. The goal is to identify in real time $\Delta$ and $\mu$, measuring the ground state population thanks to the first laser (which generates a transition only for ground state population) and the photo-detector. The usual modeling of these open-quantum systems via Lindbald type terms in the density matrix dynamics (see, e.g., Haroche and Raimond [2006]) is analyzed in Mirrahimi and Rouchon [2006], where singular perturbation techniques are applied to justify the adiabatic (quasi-static) approximations usually made by physicists and leading to a dynamical model described (up to higher order terms) by a Schrödinger equation despite a continuous population measurement.

In this paper we build an observer which estimates the wave function (in fact we rather use the density matrix language) and the two parameters $\Delta$ and $\mu$ at the same time. We propose an extension of the observer described in Mirrahimi and Rouchon [2007], where only $\mu$ was estimated, and we show that symmetries (invariance by change of frame described by an element of $S U(2)$ ) play an important role. We use the standard notations in quantum physics literature (see e.g. Cohen-Tannoudji et al. 
[1977], and Haroche and Raimond [2006] for a more advanced lesson).

\section{DYNAMICS AND INVARIANCES}

\subsection{Dynamics}

The Schrödinger equation for the system writes:

$$
\imath \frac{d}{d t} \Psi=\left(\frac{\Delta}{2} \sigma_{z}+\frac{u \mu}{2} \sigma_{x}\right) \Psi, \quad \Psi=\left(\begin{array}{l}
\Psi_{1} \\
\Psi_{2}
\end{array}\right) \in \mathbb{C}^{2}
$$

where we let

$$
\sigma_{x}=\left(\begin{array}{ll}
0 & 1 \\
1 & 0
\end{array}\right), \quad \sigma_{y}=\left(\begin{array}{cc}
0 & -i \\
i & 0
\end{array}\right), \quad \sigma_{z}=\left(\begin{array}{cc}
1 & 0 \\
0 & -1
\end{array}\right)
$$

denote the Pauli matrices, $\Delta$ is the difference between the atomic frequency transition (of ground state to excited state $\left.\omega_{e 0}=\omega_{a, e}-\omega_{a, 0}\right)$ and the laser frequency $\omega, \mu$ is the atomlaser coupling constant and $u(t) \in \mathbb{R}$ is the slowly varying amplitude of the laser. We have the useful foumlas $\sigma_{x}^{2}=1$; $\sigma_{x} \sigma_{y}=\imath \sigma_{z}$ (with circular permutation), the output is

$$
y=<\sigma_{z} \Psi, \Psi>=\left|\Psi_{1}\right|^{2}-\left|\Psi_{2}\right|^{2}=2\left|\Psi_{1}\right|^{2}-1
$$

as the measurement is the ground state population $\left|\Psi_{1}\right|^{2}$ and the conservation of probability implies $\left|\Psi_{1}\right|^{2}+\left|\Psi_{2}\right|^{2}=1$. For a justification (in the frame of singular perturbation theory) of this model (applying weak measurement) see Mirrahimi and Rouchon [2006].

It is convenient to write the dynamics with the density matrix: let $\rho=\Psi \Psi^{\dagger}$ denote the complex matrix associated to the projector on the state $\Psi$. Supposing that the system is pure (meaning it is not entangled to its environment) implies both properties $\operatorname{Tr}(\rho)=\Psi_{1}^{\dagger} \Psi_{1}+\Psi_{2}^{\dagger} \Psi_{2}=1$ and $\rho^{2}=\Psi \Psi^{\dagger} \Psi \Psi^{\dagger}=\rho$. Thus rewriting (1) the system becomes

$$
\begin{aligned}
\dot{\rho} & =-l\left[\frac{\Delta}{2} \sigma_{z}+\frac{u \mu}{2} \sigma_{x}, \rho\right] \\
\dot{\mu} & =0 \\
\dot{\Delta} & =0 \\
y & =\operatorname{Tr}\left(\sigma_{z} \rho\right)
\end{aligned}
$$

where [,] is the commutator. We assume the laser amplitude to be slowly varying compared to the Rabi frequency $|u \mu|$ : $|\dot{u}|<<|u \mu||u|$ (the Rabi frequency is a characteristic of the absorption-emission cycle of photons for an illuminated atom). We assume, moreover, that that the frequencies of the laser and the frequencies of the atomic transition are close (i.e, smaller than the atomic width): $|\Delta|<<|u| \mu$.

\subsection{Invariances (symmetries)}

The system is invariant under a change of basis for the wave function $\Psi \mapsto U \Psi$ where $U$ is any unit matrix of the Lie group $S U(2)$. Indeed consider the transformation $\varpi=U \rho U^{\dagger}$, and $\zeta_{x}=U \sigma_{x} U^{\dagger}, \zeta_{y}=U \sigma_{y} U^{\dagger}, \zeta_{z}=U \sigma_{z} U^{\dagger}$. With the new variables, the dynamics (2)-(5) writes

$$
\begin{aligned}
\frac{d}{d t} \varpi & =-\imath\left[\frac{\Delta}{2} \zeta_{z}+\frac{u \mu}{2} \zeta_{x}, \varpi\right] \\
y & =\operatorname{Tr}\left(\zeta_{z} \varpi\right)
\end{aligned}
$$

$\Delta$ and $\mu$ are unchanged by the transformation and we still have $\dot{\Delta}=\dot{\mu}=0 . \zeta_{x}, \zeta_{y}, \zeta_{z}$ respect the commutation relations of the Pauli matrices. Thus the system is invariant under the action of the transformation group $S U(2)$ (see definition 2 of section 7).

\subsection{Invariants, Invariant output, invariant vector fields}

We are going to explain the form of the (symmetry-preserving) observer (6)-(7)-(8)-(9). For simplicity's sake in this section we consider the reduced system (2)-(5)

$$
\begin{aligned}
\dot{\rho} & =-\imath\left[\frac{\Delta}{2} \sigma_{z}+\frac{u \mu}{2} \sigma_{x}, \rho\right] \\
y & =\operatorname{Tr}\left(\sigma_{z} \rho\right)
\end{aligned}
$$

since the group only acts on $\rho$ and not on $\Delta$ and $\mu$. To find the form of observers which preserve the symmetries (i.e, are invariant under the action of $S U(2)$, see section 7), we follow the method of Mirrahimi and Rouchon [2007]. Even if the method does not fully apply to the two-state quantum system under study (all the results are local and the dimension of the group $r$ must be strictly smaller than the dimension of the state space $n$ ), it gives guidelines which help us build a symmetry-preserving observer. We recall some basic definitions and results of this latter paper in the appendix (section 7). In particular, we need to build an invariant output error, an invariant frame, and scalar invariants of the group action (see eq (24) of section 7).

Invariant output error: The output $y=\operatorname{Tr}\left(\sigma_{z} \rho\right)$ is a scalar. It is invariant under the group action since for any $U \in S U(2)$ if we let $\varpi=U \rho U^{\dagger}$ and $\zeta_{z}=U \sigma_{z} U^{\dagger}$ we have $y=\operatorname{Tr}\left(\zeta_{z} \varpi\right)=$ $\operatorname{Tr}\left(U \sigma_{z} \rho U^{\dagger}\right)=\operatorname{Tr}\left(\sigma_{z} \rho\right)$. Thus an output error is (see the definition 5 of section 7) : $\hat{y}-y=\operatorname{Tr}\left(\sigma_{z}(\hat{\rho}-\rho)\right)$.

Invariant vector fields: The system (2) is invariant thus the second member of (2) is made of invariant vector fields (in the sense of the definition 3 of section 7). Inspiring from the form of the dynamics, let us take as invariant vector fields $-l\left[\sigma_{x}, \cdot\right]$, ${ }_{-l}\left[\sigma_{y}, \cdot\right]$ and $-l\left[\sigma_{z}, \cdot\right]$. They provide a global parameterization of the tangent bundle of the space of $2 \times 2$ projector matrices with trace 1 . Notice that, they are functionally dependant.

Scalar invariants: A complete set of scalar invariants is a full rank function $\left(\hat{\rho}, \sigma_{x}, \sigma_{y}, \sigma_{z}\right) \mapsto I\left(\hat{\rho}, \sigma_{x}, \sigma_{y}, \sigma_{z}\right) \in \mathbb{R}^{n+m-r}$ which is invariant under the group action (where $n$ is the dimension of the state space, $m$ is the dimension on the inputs on which $\left(\psi_{g}\right)_{g \in G}$ act, and $r$ is the dimension of the group $\left.\mathrm{G}\right)$. Here $n=2, m=3$ and $r=3$. Locally there are $n+m-r=2+3-$ $3=2$ scalar invariants (see Olver [1995]). In fact we take the 3 invariants: $\operatorname{Tr}\left(\sigma_{x} \hat{\rho}\right), \operatorname{Tr}\left(\sigma_{y} \hat{\rho}\right), \operatorname{Tr}\left(\sigma_{z} \hat{\rho}\right)$. They are functionally dependent since $\operatorname{Tr}^{2}\left(\sigma_{x} \hat{\rho}\right)+\operatorname{Tr}^{2}\left(\sigma_{y} \hat{\rho}\right)+\operatorname{Tr}^{2}\left(\sigma_{z} \hat{\rho}\right)=1$. (see section 3.2).

\section{A SYMMETRY-PRESERVING OBSERVER}

\subsection{The observer}

Consider the observer

$$
\begin{aligned}
\frac{d}{d t} \hat{\rho}= & -\imath\left[\frac{\hat{\Delta}}{2} \sigma_{z}+\frac{u \hat{\mu}}{2} \sigma_{x}, \hat{\rho}\right] \\
& -K_{\rho}\left(\operatorname{Tr}\left(\sigma_{z} \hat{\rho}\right)-y\right)\left(\sigma_{z} \hat{\rho}+\hat{\rho} \sigma_{z}-2 \operatorname{Tr}\left(\sigma_{z} \hat{\rho}\right) \hat{\rho}\right) \\
\frac{d}{d t} \hat{\mu}= & -u K_{\mu} \operatorname{Tr}\left(\sigma_{y} \hat{\rho}\right)\left(\operatorname{Tr}\left(\sigma_{z} \hat{\rho}\right)-y\right) \\
\frac{d}{d t} \hat{\Delta}= & -u K_{\Delta} \operatorname{Tr}\left(\sigma_{x} \hat{\rho}\right)\left(\operatorname{Tr}\left(\sigma_{z} \hat{\rho}\right)-y\right)
\end{aligned}
$$

where $K_{\rho}, K_{\mu}$ and $K_{\Delta}$ are positive scalars. It preserves the symmetries (see eq (24) of section 7) since (7) can be written (see section 3.2 for the proof) 


$$
\begin{aligned}
\frac{d}{d t} \hat{\rho}= & -l\left[\frac{\hat{\Delta}}{2} \sigma_{z}+\frac{u \hat{\mu}}{2} \sigma_{x}, \hat{\rho}\right] \\
& +\imath K_{\rho}\left(\operatorname{Tr}\left(\sigma_{z} \hat{\rho}\right)-y\right)\left(\operatorname{Tr}\left(\sigma_{y} \hat{\rho}\right)\left[\sigma_{x}, \hat{\rho}\right]-\operatorname{Tr}\left(\sigma_{x} \hat{\rho}\right)\left[\sigma_{y}, \hat{\rho}\right]\right)
\end{aligned}
$$

As we did for the true system, let us suppose that $u$ is constant, and $|\hat{\Delta}| \approx \varepsilon|u \mu|$. To be able to apply the standard perturbation techniques for this type of physical system we choose the gains

$$
K_{\rho}=4 k_{\rho} \varepsilon|u| \mu, K_{\mu}=2 k_{\mu} \varepsilon^{2} \mu^{2}, K_{\Delta}=2 k_{\Delta} \varepsilon^{2}|u| \mu^{2}
$$

with $\varepsilon>0$ small $(\varepsilon \ll 1)$, and $k_{\rho}, k_{\mu}, k_{\Delta} \sim 1$.

\subsection{Geometrical interpretation with the Bloch sphere}

The Bloch sphere is a geometrical representation of the pure state space of a two-level quantum mechanical system. An important property is that any density matrix $\rho$ can be written

$$
\rho=\frac{1+X \sigma_{x}+Y \sigma_{y}+Z \sigma_{z}}{2}, \text { with } \zeta=\left(\begin{array}{l}
X \\
Y \\
Z
\end{array}\right) \in \mathbb{S}^{2}
$$

where 1 denotes the identity $2 \times 2$ matrix. We have $\operatorname{Tr}\left(\sigma_{x} \rho\right)=$ $X, \operatorname{Tr}\left(\sigma_{y} \rho\right)=Y$ and $\operatorname{Tr}\left(\sigma_{z} \rho\right)=Z$. Note that, the coordinate $Z$ in this section is the output $y$. The commutation operation $-l\left[\sigma_{x}, \rho\right]$ corresponds to the wedge product $(1,0,0)^{T}$ with $(X, Y, Z)^{T}$ (circular permutations allow to complete the correspondences). The dynamics of $\zeta$ is

$$
\frac{d}{d t} \zeta=\left(\frac{u \mu}{2}, 0, \frac{\Delta}{2}\right)^{T} \wedge \zeta
$$

The dynamics does not depend on the choice of the orthonormal frame (invariance under the action of $S O(3)$ ).

The correction term in (7) writes $-K_{\rho}(\hat{Z}-Z)\left[-\hat{X} \hat{Z} \sigma_{x}-\right.$ $\left.\hat{Y} \hat{Z} \sigma_{y}+\left(1-\hat{Z}^{2}\right) \sigma_{z}\right]$ and corresponds on the Bloch sphere to $-K_{\rho}(\hat{Z}-Z)(\hat{Y},-\hat{X}, 0)^{T} \wedge \hat{\zeta}$, where $\hat{\zeta}=(\hat{X}, \hat{Y}, \hat{Z})^{T}$. It respects the symmetries (invariance by change of orthonormal frame) since it bounds $\zeta$ to remain on $\mathbb{S}^{2}$ (meaning physically that the system is pure).

Choice of the invariant correction terms This paragraph explains the geometrical motivations of the choice of the gains. More rigorous proofs are given in the next section. The correction term of (7) is such that it is a tangent vector to the sphere (so $\zeta$ remains in $\mathbb{S}^{2}$ ) and its direction is such that it tends to make output error $\hat{Z}-Z$ decrease. Indeed the dynamics of $\hat{\zeta}$ is:

$$
\frac{d}{d t} \hat{\zeta}=\frac{1}{2}\left(\begin{array}{c}
u \hat{\mu} \\
0 \\
\hat{\Delta}
\end{array}\right) \times \hat{\zeta}-K_{\rho}(\hat{Z}-Z)\left(\hat{\zeta} \times\left(\begin{array}{l}
0 \\
0 \\
1
\end{array}\right)\right) \times \hat{\zeta}
$$

But

$$
<\left(\hat{\zeta} \times\left(\begin{array}{l}
0 \\
0 \\
1
\end{array}\right)\right) \times \hat{\zeta},\left(\begin{array}{l}
0 \\
0 \\
1
\end{array}\right)>=<\hat{\zeta} \times\left(\begin{array}{l}
0 \\
0 \\
1
\end{array}\right), \hat{\zeta} \times\left(\begin{array}{l}
0 \\
0 \\
1
\end{array}\right)>\geq 0
$$

is always positive (where $<,>$ denotes the usual scalar product). The correction term is a vector which is in fact always pointing towards "north" (i.e, $Z=1$ ) if $\hat{Z}<Z$ and towards "south" (i.e, $Z=-1$ ) if not.

$K_{\rho}$ is much bigger than $K_{\mu}$ and $K_{\Delta}$, meaning that the time scale of the convergence of $\hat{Z}$ towards $Z$ is faster. Let us thus suppose $\hat{Z}-Z$ is close to 0 . If $u \hat{\mu}>u \mu$, then $\hat{\zeta}$ tends to rotate faster around the $X$-axis than $\zeta$, and since $\frac{d}{d t} Z=u \mu Y$, the difference $\hat{Z}-Z$ tends to increase if $Y>0$ and decrease if not. That explains (8) which also writes $\frac{d}{d t} \hat{\mu}=-u K_{\mu} \hat{Y}(\hat{Z}-Z)$. The design (9) can be explained in the same way.

\section{CONVERGENCE ISSUE}

In this section we prove the existence of a Lyapunov function for the reduced system (after having performed an averaging of the periodic perturbations). To apply the rotating wave approximation one writes the system in the interaction frame, i.e, one makes the time dependant change of variables:

$$
\rho=e^{-l \frac{u \mu t \sigma_{x}}{2}} \xi e^{l \frac{u \mu t \sigma_{x}}{2}}, \quad \hat{\rho}=e^{-l \frac{u \mu t \sigma_{x}}{2}} \hat{\xi} e^{l \frac{u \mu t \sigma_{x}}{2}} .
$$

We have thus

$$
\frac{d}{d t} \xi=\left[l \frac{u \mu \sigma_{x}}{2}, \xi\right]+e^{l \frac{u \mu t \sigma_{x}}{2}}\left(\frac{d}{d t} \rho\right) e^{-l \frac{u \mu t \sigma_{x}}{2}}
$$

and one can derive a similar formula for $\frac{d}{d t} \hat{\xi}$. Thus

$$
\frac{d}{d t} \xi=-l\left[\frac{\Delta}{2} e^{l u \mu t \sigma_{x}} \sigma_{z}, \xi\right]
$$

which shows the interest of the interaction frame. Note that $\sigma_{z} \sigma_{x}^{2 k}=\sigma_{x}^{2 k} \sigma_{z}$ and $\sigma_{z} \sigma_{x}^{2 k+1}=-\sigma_{x}^{2 k+1} \sigma_{z}$ for any $k$, and these relations are also true when $\sigma_{z}$ is replaced by $\sigma_{y}$ thus

$$
\sigma_{z} e^{l \frac{u \mu t \sigma_{x}}{2}}=e^{-l \frac{u \mu t \sigma_{x}}{2}} \sigma_{z}, \quad \sigma_{y} e^{l \frac{u \mu t \sigma_{x}}{2}}=e^{-l \frac{u \mu t \sigma_{x}}{2}} \sigma_{y} .
$$

So the observer, in the interaction frame, reads:

$$
\begin{aligned}
\frac{d}{d t} \hat{\xi}= & -\imath\left[\frac{\hat{\Delta}}{2} e^{i u \mu t \sigma_{x}} \sigma_{z}+\frac{u(\hat{\mu}-\mu)}{2} \sigma_{x}, \hat{\xi}\right] \\
- & K_{\rho} \operatorname{Tr}\left(e^{\imath u \mu t \sigma_{x}} \sigma_{z}(\hat{\xi}-\xi)\right) \times \\
& \left(e^{\imath u \mu t \sigma_{x}} \sigma_{z} \hat{\xi}+\hat{\xi} e^{\imath u \mu t \sigma_{x}} \sigma_{z}-2 \operatorname{Tr}\left(e^{\imath u \mu t \sigma_{x}} \sigma_{z} \hat{\xi}\right) \hat{\xi}\right) \\
\frac{d}{d t} \hat{\mu}= & -u K_{\mu} \operatorname{Tr}\left(e^{\imath u \mu t \sigma_{x}} \sigma_{y} \hat{\xi}\right) \operatorname{Tr}\left(e^{\imath u \mu t \sigma_{x}} \sigma_{z}(\hat{\xi}-\xi)\right) \\
\frac{d}{d t} \hat{\Delta}= & -u K_{\Delta} \operatorname{Tr}\left(\sigma_{x} \hat{\xi}\right) \operatorname{Tr}\left(e^{\imath u \mu t \sigma_{x}} \sigma_{z}(\hat{\xi}-\xi)\right) .
\end{aligned}
$$

Secular first-order approximation Also called quasi-resonant, it consists in neglecting the terms rotating with high frequencies $u \mu$ and $2 u \mu$, by averaging their influence on the evolution of $\rho$ (see, e.g., Haroche and Raimond [2006] for a physicist point of view on the standard rotating wave approximation or Arnold [1976] for a more formal exposure). The true dynamics consists of small oscillations around the solution of the averaged system. In order to compute the averaged system we use for $a>0$

$$
e^{l a \sigma_{x}} \sigma_{z}=\sigma_{z}+a \sigma_{y}-\frac{a^{2}}{2} \sigma_{z}-\cdots=\cos (a) \sigma_{z}+\sin (a) \sigma_{y}
$$

and similarly

$$
e^{l a \sigma_{x}} \sigma_{y}=\cos (a) \sigma_{y}-\sin (a) \sigma_{z}
$$

We write the cosine and sine as linear combinations of exponentials and we use interference formulas of the type:

$$
\begin{aligned}
& \operatorname{Tr}\left(e^{i u \mu t \sigma_{x}} \sigma_{z}(\hat{\xi}-\xi)\right) e^{i u \mu t \sigma_{x}} \sigma_{z} \hat{\xi} \\
& =\frac{1}{4}\left(2 \operatorname{Tr}\left(\sigma_{z}(\hat{\xi}-\xi)\right) \sigma_{z} \hat{\xi}+0+2 \operatorname{Tr}\left(\sigma_{y}(\hat{\xi}-\xi)\right) \sigma_{y} \hat{\xi}+0\right) \\
& \quad+\text { oscillating terms with mean } 0
\end{aligned}
$$

We therefore get the following autonomous system for the averaged system/observer: 


$$
\begin{array}{r}
\frac{d}{d t} \xi=0, \quad \frac{d}{d t} \mu=0, \quad \frac{d}{d t} \Delta=0 \\
\frac{d}{d t} \hat{\xi}=-\imath\left[\frac{u(\hat{\mu}-\mu)}{2} \sigma_{x}, \hat{\xi}\right] \\
-\frac{K_{\rho}}{2} \operatorname{Tr}\left(\sigma_{y}(\hat{\xi}-\xi)\right)\left(\sigma_{y} \hat{\xi}+\hat{\xi} \sigma_{y}-2 \operatorname{Tr}\left(\sigma_{y} \hat{\xi}\right) \hat{\xi}\right) \\
-\frac{K_{\rho}}{2} \operatorname{Tr}\left(\sigma_{z}(\hat{\xi}-\xi)\right)\left(\sigma_{z} \hat{\xi}+\hat{\xi} \sigma_{z}-2 \operatorname{Tr}\left(\sigma_{z} \hat{\xi}\right) \hat{\xi}\right)
\end{array}
$$

$\frac{d}{d t} \hat{\Delta}=0$

Lemma 1. For all $K_{\rho}, K_{\mu}>0, \hat{\xi}$ and $\hat{\mu}$ converge locally towards $\xi$ and $\mu$ (c.f. Mirrahimi and Rouchon [2007]).

Proof. We consider the Lyapunov function

$$
V=\frac{1}{2} \operatorname{Tr}^{2}\left(\sigma_{y}(\hat{\xi}-\xi)\right)+\frac{1}{2} \operatorname{Tr}^{2}\left(\sigma_{z}(\hat{\xi}-\xi)\right)+\frac{1}{K_{\mu}}(\hat{\mu}-\mu)^{2}
$$

Note that $\operatorname{Tr}\left(\frac{d}{d t} \sigma_{y}(\hat{\xi}-\xi)\right)=-\frac{u(\hat{\mu}-\mu)}{2} \operatorname{Tr}\left(2 \sigma_{z} \hat{\xi}\right)+\cdots$ and $\operatorname{Tr}\left(\frac{d}{d t} \sigma_{z}(\hat{\xi}-\xi)\right)=\frac{u(\hat{\mu}-\mu)}{2} \operatorname{Tr}\left(2 \sigma_{y} \hat{\xi}\right)+\cdots$. When developing $\frac{d}{d t} V$ the terms having $u$ as a factor compensate each other and there only remain the terms with $K_{\rho}$ as a factor. Let $\delta \xi$ denote $\hat{\xi}-\xi$. We have

$$
\begin{aligned}
\frac{d V}{d t}= & -K_{\rho} \operatorname{Tr}^{2}\left(\sigma_{y} \delta \xi\right)\left(1-\operatorname{Tr}^{2}\left(\sigma_{y} \hat{\xi}\right)\right) \\
& -K_{\rho} \operatorname{Tr}^{2}\left(\sigma_{z} \delta \xi\right)\left(1-\operatorname{Tr}^{2}\left(\sigma_{z} \hat{\xi}\right)\right) \\
& +2 K_{\rho} \operatorname{Tr}\left(\sigma_{y} \delta \xi\right) \operatorname{Tr}\left(\sigma_{z} \delta \xi\right) \operatorname{Tr}\left(\sigma_{y} \hat{\xi}\right) \operatorname{Tr}\left(\sigma_{z} \hat{\xi}\right) \\
\leq & -K_{\rho}\left(\operatorname{Tr}\left(\sigma_{y} \delta \xi\right) \operatorname{Tr}\left(\sigma_{z} \hat{\xi}\right)-\operatorname{Tr}\left(\sigma_{z} \delta \xi\right) \operatorname{Tr}\left(\sigma_{y} \hat{\xi}\right)\right)^{2} \\
\leq & 0 .
\end{aligned}
$$

Here we have used the fact that $\operatorname{Tr}^{2}\left(\sigma_{x} \hat{\xi}\right)+\operatorname{Tr}^{2}\left(\sigma_{y} \hat{\xi}\right)+$ $\operatorname{Tr}^{2}\left(\sigma_{z} \hat{\xi}\right)=1$. See Mirrahimi and Rouchon [2007] for the end of the proof which is a standard application of the Lasalle principle.

Second order secular approximation Since the secular (nonoscillating) first-order terms vanish when computing $\frac{d}{d t} \xi$ and $\frac{d}{d t} \hat{\Delta}$, one can not prove the convergence of the observer. One needs to compute the second-order approximation only for $\frac{d}{d t} \xi$ and $\frac{d}{d t} \hat{\Delta}$. We apply the Kapitsa method described in e.g, Landau and Lifshitz [1982]. Suppose $u \mu=v$ is large. $\xi$ obeys a differential equation with a high frequency source term $\frac{d}{d t} \xi=$ $f(\xi, v t)$. We proved that the mean of $\xi$ over a period is constant. Integrating high frequency terms yields high frequency terms with same frequency and smaller amplitude: we seek a solution of the type

$$
\xi=\zeta+\frac{g_{1}(\zeta, t)}{v}+\frac{g_{2}(\zeta, t)}{v^{2}}+\cdots
$$

where $\zeta$ is the mean of $\xi$ over a period (recall $\dot{\zeta}=0+O(1 / v)$ ). Let us compute the first-order term, $g_{1}(\zeta, t)$, and neglect the second-order terms. On one hand, we have:

$$
\frac{d}{d t} \xi=0+\frac{g_{1}^{\prime}(\zeta, t)}{v}
$$

where $g_{1}^{\prime}=\partial_{2} g_{1}$ is the partial derivative of $g_{1}$ with respect to its second variable. But using (10) and neglecting third order terms:

$$
\frac{d}{d t} \xi=-\imath\left[\frac{\Delta}{2} e^{i v t \sigma_{x}} \sigma_{z}, \zeta+\frac{g_{1}(\zeta, t)}{v}\right] .
$$

Gathering the two latter equations

$$
g_{1}^{\prime}(\zeta, t)=-v i\left[\frac{\Delta}{2} e^{i v t \sigma_{x}} \sigma_{z}, \zeta\right]+\cdots
$$

where we have not written the small terms compared to $v$. Integrating with respect to time $t$ the last equation yields:

$$
g_{1}(\zeta, t)=\imath\left[\frac{\Delta}{2} e^{i v t \sigma_{x}} \sigma_{y}, \xi\right]+\frac{1}{v} \cdots .
$$

Thus (14) can be re-written as

$$
\begin{aligned}
\frac{d}{d t} \xi & =-l\left[\frac{\Delta}{2} e^{i v t \sigma_{x}} \sigma_{z}, \zeta+g_{1}(\zeta, t) / v\right]+\cdots \\
& =-l\left[\frac{\Delta}{2} e^{i v t \sigma_{x}} \sigma_{z}, \zeta\right]+\frac{\Delta^{2}}{4 v}\left[e^{i v t \sigma_{x}} \sigma_{z},\left[e^{i v t \sigma_{x}} \sigma_{y}, \xi\right]\right]+\cdots .
\end{aligned}
$$

Now let us compute the temporal mean (over a period) and only keep the secular terms. We apply (12) and (13). Applying the Jacobi identity

$$
\frac{d}{d t} \xi=-l \frac{\Delta^{2}}{2 u \mu}\left[\sigma_{x}, \xi\right]+\cdots
$$

where we have not written the oscillating terms of 0 mean nor the terms of order $O\left(\frac{1}{v^{2}}\right)$. Note that, here, we find the term $\frac{\Delta^{2}}{2 u \mu}$ corresponding to the standard Bloch-Siegert shift.

We also need to develop $\hat{\Delta}$ up to second order terms (coefficients of $\frac{1}{v}$ ) since it has constant mean. Its time derivative verifies

$$
\frac{d}{d t} \hat{\Delta}=-u K_{\Delta} \operatorname{Tr}\left(\sigma_{x} \hat{\xi}\right) \operatorname{Tr}\left(e^{\imath u \mu t \sigma_{x}} \sigma_{z}(\hat{\xi}-\xi)\right)
$$

Note that, $\xi$ and $\hat{\xi}$ are themselves solutions of differential equations depending on $t$ via oscillating terms of frequency $v$. they have an oscillating part of frequency $v$ and another one of frequency $2 v$, since their time derivatives are sum and products of at most two oscillating terms of frequency $v$. When considering up to the second order, only the terms of frequency $v$ in $\xi$ and $\hat{\xi}$ can have a secular effect, as they multiply the $v$-frequency term $e^{i u \mu t \sigma_{x}} \sigma_{z}$. Thus in this second order secular approximation the oscillating terms of frequency $2 v$ can be neglected.

But, the $v$-frequency part of $\hat{\xi}$ is due to the integration of $-l\left[\frac{\hat{\Delta}}{2} e^{\imath u \mu t \sigma_{x}} \sigma_{z}, \hat{\xi}\right]$ and so is for $\xi$. Thus

$$
\begin{aligned}
& \hat{\xi}=\hat{\zeta}+\frac{\imath \hat{\Delta}}{2 u \mu}\left[e^{u l \mu t \sigma_{x}} \sigma_{y}, \hat{\xi}\right]+\cdots \\
& \xi=\zeta+\frac{\imath \Delta}{2 u \mu}\left[e^{i u \mu t \sigma_{x}} \sigma_{y}, \xi\right]+\cdots
\end{aligned}
$$

where $\hat{\zeta}$ (resp $\zeta$ ) is a solution of the averaged equation for $\hat{\xi}$ (resp $\hat{\xi}$ ) and the non-written terms are either $2 v$-frequency terms or are of order $O\left(\frac{1}{v^{2}}\right)$. Up to the second order, the secular terms of (15) can be calculated as the sum of two parts: 1 . replacing $\hat{\xi}-\xi$ by its $v$-frequency part in order to compute the secular terms of $\operatorname{Tr}\left(e^{u \mu t \sigma_{x}} \sigma_{z}(\hat{\xi}-\xi)\right)$; this will be then multiplied by $-u K_{\Delta} \operatorname{Tr}\left(\sigma_{x} \hat{\xi}\right) ; 2$. replacing $\hat{\xi}$ in $\operatorname{Tr}\left(\sigma_{x} \hat{\xi}\right)$ by 
its $v$-frequency terms, leaving $\hat{\xi}-\xi$ as it is and calculating the secular terms of $\operatorname{Tr}\left(\sigma_{x} \hat{\xi}\right) \operatorname{Tr}\left(e^{i l \mu t \sigma_{x}} \sigma_{z}(\hat{\xi}-\xi)\right)$.

On one hand, we have

$$
\operatorname{Tr}\left(e^{\imath v t \sigma_{x}} \sigma_{z}(\hat{\xi}-\xi)\right)=\frac{1}{v}\left(\hat{\Delta} \operatorname{Tr}\left(\sigma_{x} \hat{\xi}\right)-\Delta \operatorname{Tr}\left(\sigma_{x} \xi\right)\right)+\cdots
$$

where we have not written the oscillating terms of 0 mean. Here, we have applied (16) and the following relation:

$$
\imath \frac{\hat{\Delta}}{2 v} e^{\imath v t \sigma_{x}} \sigma_{z}\left[e^{\imath v t \sigma_{x}} \sigma_{y}, \hat{\xi}\right]=\imath \frac{\hat{\Delta}}{2 v}\left(\sigma_{z}\left[\sigma_{y}, \hat{\xi}\right]-\imath \sigma_{y}\left[\sigma_{y}, \hat{\xi}\right]\right)+\cdots
$$

On the other hand, for the $v$-frequency part of $\operatorname{Tr}\left(\sigma_{x} \hat{\xi}\right)$, using (16), we have:

$$
\begin{aligned}
\operatorname{Tr}\left(\sigma_{x}\left[e^{\imath v t \sigma_{x}} \sigma_{y}, \hat{\xi}\right]\right) & =\operatorname{Tr}\left(\cos v t \sigma_{x}\left[\sigma_{y}, \hat{\xi}\right]-\sin v t \sigma_{x}\left[\sigma_{z}, \hat{\xi}\right]\right) \\
& =2 \imath \operatorname{Tr}\left(\cos v t \sigma_{z} \hat{\xi}+\sin v t \sigma_{y} \hat{\xi}\right)
\end{aligned}
$$

Therefore, up to the second order, we have

$$
\begin{aligned}
\frac{d}{d t} \hat{\Delta}= & -\frac{K_{\Delta}}{\mu}\left(\operatorname{Tr}\left(\sigma_{x} \hat{\xi}\right)^{2} \hat{\Delta}-\operatorname{Tr}\left(\sigma_{x} \hat{\xi}\right) \operatorname{Tr}\left(\sigma_{x} \xi\right) \Delta\right) \\
& +\frac{K_{\Delta} \hat{\Delta}}{2 \mu}\left[\operatorname{Tr}\left(\sigma_{y} \hat{\xi}\right) \operatorname{Tr}\left(\sigma_{y}(\hat{\xi}-\xi)\right)\right. \\
& \left.-\operatorname{Tr}\left(\sigma_{z} \hat{\xi}\right) \operatorname{Tr}\left(\sigma_{z}(\hat{\xi}-\xi)\right)\right] .
\end{aligned}
$$

We have obtained the following, locally convergent, triangular system:

$$
\begin{aligned}
& \frac{d}{d t} \xi \stackrel{\text { order } 2}{=}- l \frac{\Delta^{2}}{2 u \mu}\left[\sigma_{x}, \xi\right] \\
& \frac{d}{d t} \hat{\xi} \stackrel{\text { order } 1}{=}-l {\left[\frac{u(\hat{\mu}-\mu)}{2} \sigma_{x}, \hat{\xi}\right] } \\
& \quad-\frac{K_{\rho}}{2} \operatorname{Tr}\left(\sigma_{y}(\hat{\xi}-\xi)\right)\left(\sigma_{y} \hat{\xi}+\hat{\xi} \sigma_{y}-2 \operatorname{Tr}\left(\sigma_{y} \hat{\xi}\right) \hat{\xi}\right) \\
& \quad-\frac{K_{\rho}}{2} \operatorname{Tr}\left(\sigma_{z}(\hat{\xi}-\xi)\right)\left(\sigma_{z} \hat{\xi}+\hat{\xi} \sigma_{z}-2 \operatorname{Tr}\left(\sigma_{z} \hat{\xi}\right) \hat{\xi}\right) \\
& \frac{d}{d t} \hat{\mu} \stackrel{\text { order } 1}{=}-\frac{u K_{\mu}}{2}\left[\operatorname{Tr}\left(\sigma_{y} \hat{\xi}\right) \operatorname{Tr}\left(\sigma_{z}(\hat{\xi}-\xi)\right)\right.\left.-\operatorname{Tr}\left(\sigma_{z} \hat{\xi}\right) \operatorname{Tr}\left(\sigma_{y}(\hat{\xi}-\xi)\right)\right] \\
& \frac{d}{d t} \hat{\Delta} \stackrel{\text { order } 2}{=}-\frac{K_{\Delta}}{\mu}\left(\operatorname{Tr}\left(\sigma_{x} \hat{\xi}\right)^{2} \hat{\Delta}-\operatorname{Tr}\left(\sigma_{x} \hat{\xi}\right) \operatorname{Tr}\left(\sigma_{x} \xi\right) \Delta\right) \\
&+\frac{K_{\Delta} \hat{\Delta}}{2 \mu}\left[\operatorname{Tr}\left(\sigma_{y} \hat{\xi}\right) \operatorname{Tr}\left(\sigma_{y}(\hat{\xi}-\xi)\right)\right. \\
&\left.\quad-\operatorname{Tr}\left(\sigma_{z} \hat{\xi}\right) \operatorname{Tr}\left(\sigma_{z}(\hat{\xi}-\xi)\right)\right] .
\end{aligned}
$$

Tuning the gains for the linearized system The lemma 1 proves (after using averaging arguments) that $\hat{\mu}-\mu$ and $\hat{\xi}-\xi$ converge (locally) to 0 for any $K_{\rho}, K_{\mu}>0$. The last equation of (17) shows that once $\hat{\xi}-\xi$ has converged to $0, \hat{\Delta}-\Delta$ converges to 0 for $K_{\Delta}>0$. Nevertheless in order to get a robust observer the tuning of the gains must respect the time scales. To choose appropriate gains, we consider the first order approximation of (17) around particular equilibrium points which are such that the linearized system writes simply around these points.

Consider the linearized error variables

$$
\hat{\xi}-\xi=\frac{1+\tilde{X} \sigma_{x}+\tilde{Y} \sigma_{y}+\tilde{Z} \sigma_{z}}{2}, \quad \tilde{\mu}=\hat{\mu}-\mu, \quad \tilde{\Delta}=\hat{\Delta}-\Delta
$$

where we have $\operatorname{Tr}\left(\sigma_{x} \xi\right)=X, \operatorname{Tr}\left(\sigma_{x} \hat{\xi}\right)=\hat{X}$ and the similar formulas for $Y, Z, \hat{Y}, \hat{Z}$. Consider the linearized system around $\xi=\frac{1-\sigma_{z}}{2}$ (i.e, $Z=-1$ ). Up to second order terms, we have

$$
\frac{d}{d t}(\hat{\xi}-\xi)=\left(-u \tilde{\mu}-K_{\rho} \tilde{Y}\right) \sigma_{y} .
$$

This can be written as

$$
\begin{aligned}
& \frac{d}{d t} \tilde{X}=0 \\
& \frac{d}{d t} \tilde{Y}=-u \tilde{\mu}-K_{\rho} \tilde{Y} \\
& \frac{d}{d t} \tilde{Z}=0
\end{aligned}
$$

We also have

$$
\frac{d}{d t} \tilde{\mu}=-u K_{\mu}(0-\tilde{Y}) / 2=u K_{\mu} \tilde{Y} / 2
$$

and

$$
\frac{d}{d t} \tilde{\Delta}=\frac{K_{\Delta}}{2 \mu} \hat{\Delta} \tilde{Z}
$$

Let us now write the linearized system around the other equilibrium point $\rho=\frac{1-\sigma_{x}}{2}$ (i.e, $X=-1$ )

and

$$
\frac{d}{d t}(\hat{\xi}-\xi)=-\frac{K_{\rho}}{2}\left(\tilde{Y} \sigma_{y}+\tilde{Z} \sigma_{z}\right), \quad \frac{d}{d t} \tilde{\mu}=0
$$

$$
\frac{d}{d t} \tilde{\Delta}=-\frac{K_{\Delta}}{\mu} \tilde{\Delta}
$$

The interesting equations for the tuning are (18), (19) and (20). To respect the time scales, set $0<\varepsilon \ll 1$ and choose the gain $K_{\rho} \approx \varepsilon|u \mu|$. In this case the observer filters the high frequencies and the average of $\|\hat{\xi}-\xi\|$ tends to decrease. Choose a slower characteristic time of convergence for the parameters $\hat{\mu}$ and $\hat{\Delta}$ with respect to the characteristic time of convergence of $\hat{\xi}$

$$
K_{\rho}=2 k_{\rho} \varepsilon|u| \mu, \quad K_{\mu}=2 \varepsilon^{2} \mu^{2}, \quad K_{\Delta}=k_{\Delta} \varepsilon^{2}|u| \mu^{2}
$$

where $k_{\rho}, k_{\Delta}>0$ are any scalar of order 1 . The tuning of the gains is only made for the linearized system around particular equilibrium points. Nevertheless we think the nonlinear structure of the observer, based on the symmetries (and thus very close to the structure of the system), allows a good global behavior, as the simulations show.

\section{SIMULATIONS}

We take for the initial conditions:

$\rho_{0}=\frac{1+\cos \left(\frac{\pi}{5}\right) \sigma_{x}+\sin \left(\frac{\pi}{5}\right) \cos \left(\frac{\pi}{1.4}\right) \sigma_{y}+\sin \left(\frac{\pi}{5}\right) \sin \left(\frac{\pi}{1.4}\right) \sigma_{z}}{2}$ $\mu=1, \Delta=\frac{1}{5}, \hat{\rho}_{0}=\sigma_{x} \rho_{0} \sigma_{x}$

We choose for the control $u$ and the gains: $u=1, K_{\rho}=2 \varepsilon|u| \mu$, $K_{\mu}=2 \varepsilon^{2} \mu^{2}$ et $K_{\Delta}=2 \varepsilon^{2}|u| \mu^{2}$ with $\varepsilon=\frac{1}{5}$. The results are given by fig 1 . The measured signals were added a white gaussian noise of amplitude $20 \%$.

\section{CONCLUSION}

One could have written directly the system on the Bloch sphere with the variables $(X, Y, Z)$. But the averaging computations in the interaction frame are easier to write with Pauli matrices. Moreover, as it is proved in Mirrahimi and Rouchon [2007] 

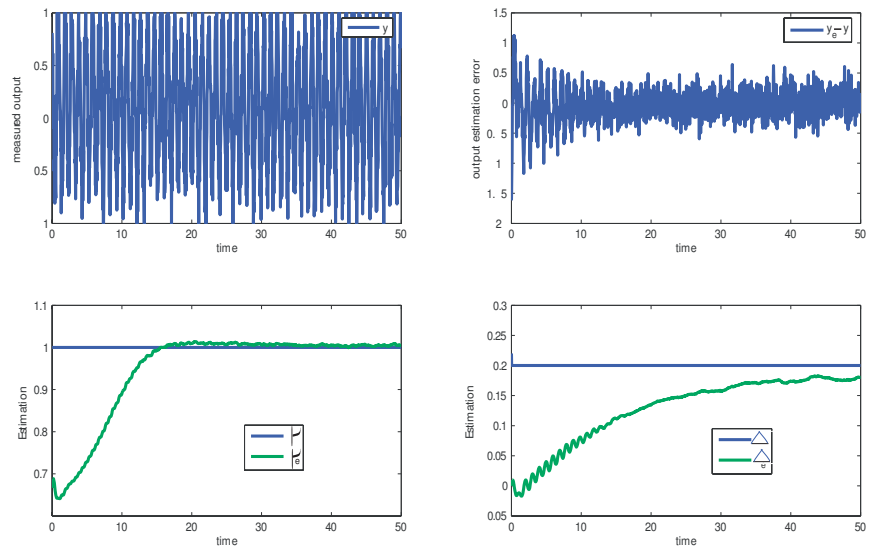

Fig. 1. Measured output, output error, estimations of the parameters $\mu$ and $\Delta$ with noisy measurement.

when $\Delta=0$, our method can be extended to more general systems for which the interpretation on the Bloch sphere is not possible anymore.

\section{APPENDIX : SYMMETRY-PRESERVING OBSERVERS}

In this section we recall the basic definitions and results of Bonnabel et al. [2006]. Consider the smooth system

$$
\begin{aligned}
\frac{d}{d t} x & =f(x, u) \\
y & =h(x, u)
\end{aligned}
$$

where $x$ belongs to an open subset $\mathscr{X} \subset \mathbb{R}^{n}, u$ to an open subset $\mathscr{U} \subset \mathbb{R}^{m}$ and $y$ to an open subset $\mathscr{Y} \subset \mathbb{R}^{p}, p \leq n$. We assume the signals $u(t), y(t)$ known. In section 2.3 we took

$$
x=\rho, \quad u=\left(\sigma_{x}, \sigma_{y}, \sigma_{z}\right), \quad y=\operatorname{Tr}\left(\sigma_{z} \rho\right)
$$

Consider also the local group of transformations on $\mathscr{X} \times \mathscr{U}$ defined by

$$
(X, U)=\left(\varphi_{g}(x), \psi_{g}(u)\right)
$$

where $\varphi_{g}$ and $\psi_{g}$ are local diffeomorphisms depending on a parameter $g$ which is an element of a Lie group $G$ such that

- $\varphi_{e}(\xi)=\xi$ for all $\xi \in \mathscr{X}$

- $\varphi_{g_{2}}\left(\phi_{g_{1}}(\xi)\right)=\varphi_{g_{2} g_{1}}(\xi)$ for all $g_{1}, g_{2} \in G, \xi \in \mathscr{X}$.

and $\psi_{g}$ verifies similar conditions. In section 2.3 we have $G=$ $S U(2)$ and for any $U \in G$

$$
\varphi_{U}=\psi_{U}: M \in \mathbb{C}^{2 \times 2} \mapsto U M U^{\dagger}
$$

Definition 2. The system $\frac{d}{d t} x=f(x, u)$ is $G$-invariant if $f\left(\varphi_{g}(x), \psi_{g}(u)\right)=D \varphi_{g}(x) \cdot f(x, u)$ for all $g, x, u$.

The property also reads $\frac{d}{d t} X=f(X, U)$, i.e., the system remains unchanged under the transformation (23).

Definition 3. A vector field $w$ on $\mathscr{X}$ is said to be $\mathrm{G}$-invariant if the system $\frac{d}{d t} x=w(x)$ is invariant. This means $w\left(\varphi_{g}(x)\right)=$ $D \varphi_{g}(x) \cdot w(x)$ for all $g, x$.

Definition 4. An invariant frame $\left(w_{1}, \ldots, w_{n}\right)$ on $\mathscr{X}$ is a set of $\mathrm{n}$ linearly point-wise independent G-invariant vector fields, i.e $\left(w_{1}(x), \ldots, w_{n}(x)\right)$ is a basis of the tangent space to $\mathscr{X}$ at $x$.

Definition 5. The smooth map $(\hat{x}, u, y) \mapsto E(\hat{x}, u, y) \in \mathbb{R}^{p}$ is an invariant output error if

- the map $y \mapsto E(\hat{x}, u, y)$ is invertible for all $\hat{x}, u$

- $E(\hat{x}, u, h(\hat{x}, u))=0$ for all $\hat{x}, u$
- $E\left(\varphi_{g}(\hat{x}), \psi_{g}(u), h\left(\varphi_{g}(x), \psi_{g}(u)\right)\right)=E(\hat{x}, u, y)$ for all $\hat{x}, u, y$ Definition 6. (pre-observer). The system $\frac{d}{d t} \hat{x}=F(\hat{x}, u, y)$ is a pre-observer of (21)-(22) if for all $x, u F(x, u, h(x, u))=f(x, u)$.

The definition does not deal with convergence; if moreover $\hat{x}(t) \rightarrow x(t)$ as $t \rightarrow+\infty$ for every (close) initial conditions, the pre-observer is an (asymptotic) observer.

Definition 7. The pre-observer $\frac{d}{d t} \hat{x}=F(\hat{x}, u, y)$ is $G$-invariant if for all $g, \hat{x}, u, y$,

$$
F\left(\varphi_{g}(\hat{x}), \psi_{g}(u), h\left(\varphi_{g}(x), \psi_{g}(u)\right)\right)=D \varphi_{g}(\hat{x}) \cdot F(\hat{x}, u, y) .
$$

The property also reads $\frac{d}{d t} \hat{X}=F(\hat{X}, U, h(X, U))$, with $X=$ $\varphi_{g}(x), U=\psi_{g}(u)$. Assume that the output map is $G$-equivariant, which is the case for the quantum mechanical system considered in this paper since the output $y$ is a scalar invariant. Then a sufficient condition for the system $\frac{d}{d t} \hat{x}=F(\hat{x}, u, y)$ to be a Ginvariant pre-observer for the $G$-invariant system $\frac{d}{d t} x=f(x, u)$ is:

$$
F(\hat{x}, u, y)=f(\hat{x}, u)+\sum_{i=1}^{n} \mathscr{L}_{i}(I(\hat{x}, u), E(\hat{x}, u, y)) w_{i}(\hat{x})
$$

where $E$ is an invariant output error, $(\hat{x}, u) \mapsto I(\hat{x}, u) \in \mathbb{R}^{n+m-r}$ is a full-rank invariant function, the $\mathscr{L}_{i}$ 's are smooth functions such that for all $\hat{x}, \mathscr{L}_{i}(I(\hat{x}, u), 0)=0$, and $\left(w_{1}, \ldots, w_{n}\right)$ is an invariant frame. This result is a consequence of the theorem 2 of Bonnabel et al. [2006]. $I$ is called a complete set of scalar invariants and verifies $I\left(\varphi_{g}(\hat{x}), \psi_{g}(u)\right)=I(\hat{x}, u)$ for any $g \in G$.

\section{REFERENCES}

V. Arnold. Mathematical Methods of Classical Mechanics. Mir Moscou, 1976.

S. Bonnabel, Ph. Martin, and P. Rouchon. Invariant asymptotic observers. http://arxiv.org/abs/math.OC/0612193, 2006. accepted for pubblication in IEEE Automatic Control.

C. Claude Le Bris, M. Mirrahimi1, H. Rabitz4, and G. Turinici. Hamiltonian identification for quantum systems: wellposedness and numerical approaches. ESAIM: COCV, 13: 378-395, 2007.

C. Cohen-Tannoudji, B. Diu, and F. Laloë. Mécanique Quantique, volume I\& II. Hermann, Paris, 1977.

S. Haroche and J.M. Raimond. Exploring the Quantum: Atoms, Cavities and Photons. Oxford University Press, 2006.

L. Landau and E. Lifshitz. Mechanics. Mir, Moscow, 4th edition, 1982.

M. Mirrahimi and P. Rouchon. Continuous measurement of a statistic quantum ensemble. In IEEE Conference on Decision and Control, pages 2465-2470, 2006.

M. Mirrahimi and P. Rouchon. Observer-based hamiltonian identification for quantum systems, 2007. URL http://arxiv.org/abs/math-ph/0703024.

P. J. Olver. Equivalence, Invariants and Symmetry. Cambridge University Press, 1995. 